\title{
Prácticas de salud de dominación y exclusión: visiones de activistas, profesionales \\ e investigadoras sobre situación de esterilizaciones forzadas en mujeres y niñas con discapacidad en España
} Health practices of domination and exclusion: views of activists, professionals and researchers on the situation of forced sterilization of women and girls with disabilities in Spain

\author{
Andrea Yupanqui-Concha ${ }^{\mathrm{a}, \mathrm{b}}$ \\ (i) https://orcid.org/0000-0003-2698-6755 \\ E-mail: andrea.yupanquiळumag.cl \\ Cristian Aranda-Farias ${ }^{a, b}$ \\ (D) https://orcid.org/0000-0002-0151-7744 \\ E-mail: cristian.aranda®umag.cl
}

Victoria A. Ferrer-Perez ${ }^{\text {b }}$

(i) https://orcid.org/0000-0002-8096-4031

E-mail: victoria.ferrerळuib.es

aniversidad de Magallanes. Facultad de Ciencias de la Salud. Departamento de Terapia Ocupacional. Punta Arenas, Magallanes y la Antártida Chilena, Chile.

bUniversidad de las Islas Baleares. Facultad de Psicología. Departamento de Psicología. Palma, Baleares, España.

\section{Correspondência}

Andrea Yupanqui-Concha

Av. Presidente Manuel Bulnes, 1.855. Punta Arenas, Magallanes y la Antártida Chilena, Chile. CP 6210427.

\section{Resumen}

En 2017, las Naciones Unidas declaran que 38 países, entre ellos España, continuaban realizando esterilizaciones forzadas en mujeres y niñas con discapacidad, a pesar de tratarse de una práctica ampliamente condenada por diversos organismos internacionales de derechos humanos. Este estudio analizó la situación de esta práctica en España, desde la perspectiva de activistas, profesionales e investigadoras con experiencias vinculadas a este colectivo. La metodología empleada fue de tipo cualitativa basada en la Teoría Fundamentada Constructivista. Se aplicaron entrevistas semiestructuradas en profundidad a 22 informantes, que representaron a 6 comunidades autónomas del país. Las participantes identificaron un modelo de prácticas de salud de dominación y exclusión, donde la sexualidad y reproducción de mujeres con discapacidad ha sido objeto de expropiación, alienación y desprecio por parte de sistemas, estructuras y políticas diferenciadas, con escasa transferencia del marco global de derechos humanos a su realidad. La violencia sexual ha pasado inadvertida, naturalizándose por el entorno, han promovido mecanismos de exclusión social e inequidades en salud, al privarles de sus derechos humanos fundamentales. Al tratarse de una situación que se replica en diversos lugares del 
mundo, debiese considerarse un tema de relevancia para la salud pública internacional.

Palabras clave: Esterilización Forzada; Mujer con Discapacidad; Derechos Humanos; Salud Sexual y Reproductiva; Exclusión Social.

\section{Abstract}

In 2017, the United Nations declared that 38 countries, including Spain, continued to practice forced sterilizations on women and girls with disabilities, despite it being a practice widely condemned by various international human rights organizations. This study analyzed the situation of this practice in Spain, from the perspective of activists, professionals and researchers with experiences related to this group. The methodology used was qualitative, based on Constructivist Grounded Theory. Semi-structured in-depth interviews were applied to 22 informants, representing 6 autonomous communities of the country. The participants identified a model of health practices of domination and exclusion, where the sexuality and reproduction of women with disabilities has been the object of expropriation, alienation and contempt by systems, structures and differentiated policies, with little transfer of the global framework of human rights to their reality. Sexual violence has gone unnoticed, becoming naturalized by the environment, and has promoted mechanisms of social exclusion and health inequities, depriving them of their fundamental human rights. As it is a situation that is replicated in various parts of the world, it should be considered an issue of relevance for international public health.

Keywords: Forced Sterilization; Women with Disabilities; Human Rights; Sexual and Reproductive Health; Social Exclusion.

\section{Introducción}

La esterilización forzada es una práctica médica que ha sido permitida en gran parte del mundo, desde la segunda mitad del siglo XIX, y persistiendo hasta nuestros días como una de las violaciones a los derechos humanos más generalizadas a nivel mundial (OHCHR et al., 2014; Sterilization..., 2011; UN, 2017). Se ha considerado que una esterilización es forzada, forzosa o involuntaria si se ejecuta sin el completo, libre e informado consentimiento de una persona o a pesar de ser rechazado expresamente, o sin que lo sepa, o sin que se le dé la oportunidad de dar su consentimiento (Sterilization..., 2011). A nivel internacional se ha distinguido como una forma de violencia contra las mujeres (UN Women, 1996); crimen de lesa humanidad y delito grave de violencia sexual (ONU, 2003); forma de tortura, trato cruel, inhumano y degradante hacia las mujeres (UNHRC, 2008), delito y práctica criminal (Consejo de Europa, 2011). Si bien los instrumentos de derechos son categóricos con el reconocimiento de la práctica, existe una brecha entre estos y las normas legales de los distintos países involucrados, pues el acto de esterilización forzada aún no ha sido prohibido explícitamente en muchos de ellos. Evidencias sostienen que se ha ejercido en poblaciones vulnerables, tales como personas con discapacidad, pueblos indígenas y minorías étnicas, personas con VIH, personas transexuales o intersexuales, entre otras. Sin embargo, mujeres y niñas con discapacidad han sido el blanco prioritario de estos procedimientos en el mundo (OHCHR et al., 2014; Rowlands; Wale, 2019; Sifris, 2016).

Europa no ha sido una excepción. Diversos países europeos llevaron a cabo este tipo de prácticas durante la II Guerra Mundial a través de sus políticas eugenésicas, permaneciendo presentes en muchos de ellos hasta la actualidad (Albert; Szilvasi, 2017; Stansfield; Holland; Clare, 2007). Particularmente, en España este procedimiento se encuentra legalizado a partir de 1989, con la aprobación de la Ley Orgánica 3/1989 de actualización del Código Penal (España, 1989), que establecía la facultad de esterilizar a una persona sujeta a tutela de modo no punible. Esta medida fue modificada en el nuevo Código Penal, a través de la Ley Orgánica 10/1995 (España, 1995), 
que en su artículo 156 incorporó el concepto de "mayor interés” de la persona 'incapacitada'. No obstante, los mecanismos de seguimiento de la Convención sobre los Derechos de las Personas con Discapacidad de España (ONU, 2019b) y de la CEDAW (ONU, 2019a), solicitaron recientemente al Estado español la derogación del artículo 156, con el propósito de suprimir esta práctica.

Algunas cifras nacionales demuestran parcialmente esta realidad, indicando que, hasta 2016, se habría aplicado a 100 personas con discapacidad en forma anual durante los últimos 10 años, pese a que esos datos no se desagregan por sexo, y no existen datos de 2016 en adelante (Cermi; EDF, 2017).

Un estudio reciente revela que esta práctica continúa siendo un tema insuficientemente estudiado y reportado en la literatura a nivel mundial (Yupanqui; Ferrer, 2019) y que, en España, esta práctica ha sido denunciada en forma prioritaria por el activismo de la discapacidad (Arnau, 2014; Cermi; EDF, 2017). Dicho activismo describe la práctica como una forma de eugenesia sutil, aunque afirman que gran parte de la sociedad no es consciente de su existencia. Coinciden en la necesidad de impulsar investigaciones que difundan la realidad de la esterilización de personas con discapacidad en el país.

En definitiva, en España se reconoce la existencia de un procedimiento médico controvertido, que se encuentra avalado por su legislación, rechazado por organizaciones activistas y de derechos humanos, con escasa exploración en la literatura científica. Como parte de este análisis, y conforme al particular escenario existente en el país, se propuso el objetivo de describir y analizar la situación actual de la práctica de esterilización forzosa en mujeres y niñas con discapacidad en España, desde la perspectiva de mujeres representativas, objetivo central del presente artículo.

\section{Método}

En este estudio se adoptó metodología cualitativa, de tipo descriptiva-relacional de los discursos. Se seleccionó el estudio colectivo de casos, centrándose en distintas poblaciones representativas, con procesos y situaciones comunes, para favorecer la comprensión acabada del fenómeno estudiado
(Stake, 1999). Se consideró apropiado a este estudio por el interés en la variación de informantes, con el propósito de capturar diversas características de la realidad humana, distintas experiencias y contextos. Con este propósito, los criterios de inclusión del estudio fueron ser mujer, mayor de 18 años, con experiencia mínima de 1 año vinculada a mujeres y/o niñas con discapacidad (con deficiencias en sus condiciones de salud física, psíquica, intelectual, sensorial o múltiples) y formar parte de uno de los grupos de mujeres representativas: (1) activista representante de colectivos de mujeres o personas con discapacidad, (2) profesional con experiencia de trabajo con mujeres con discapacidad, (3) investigadora en temáticas de discapacidad.

Sobre el concepto discapacidad, este estudio adopta la nomenclatura propuesta por Naciones Unidas (UN, 2007), que la entiende como el resultado de la interacción entre personas con deficiencias en sus condiciones de salud (física, psíquica, intelectual, sensorial o múltiples) y las barreras contextuales, actitudinales y ambientales, que evitan su participación plena y efectiva en la sociedad en igualdad de condiciones con las demás.

Se usó el muestreo por conveniencia y bola de nieve, con la finalidad de privilegiar informantes con experiencia acerca de la práctica estudiada y por la dificultad para acceder a informantes con conocimiento del tema y voluntad de participar en el estudio. La muestra estuvo compuesta por 22 participantes. Representaron a 6 de 17 comunidades autónomas del país y sus edades van de 25 a 55 años, con un promedio de 12 años de experiencia vinculadas a mujeres con discapacidad (Tabla 1). La selección de participantes mujeres se basa en hallazgos previos que demostraron que quienes han difundido la existencia de esta práctica a nivel mundial han sido principalmente mujeres (Yupanqui; Ferrer, 2019) y, como es un problema socialmente feminizado (Cermi; EDF, 2017; OHCHR et al., 2014; UN, 2017), se precisa la producción de conocimiento científico situado desde la experiencia de mujeres involucradas en el problema estudiado, elemento clave para conocer esta realidad (Biglia; Vergés-Bosch, 2016).

Por vía telefónica y/o correo electrónico entre la investigadora principal y cada una de ellas se 
informó sobre las características generales del estudio. En el proceso destacó la alta resistencia a entregar información sobre la temática en un contexto investigativo, pues se invitó a participar a 49 mujeres, de las cuales 22 aceptaron participar. La mayoría que declinó participar en el estudio manifestó no poseer información suficiente, no tener tiempo para la entrevista o no continuaron el contacto inicialmente establecido. No se conocía previamente a las entrevistadas, lo que disminuyó el sesgo en el proceso. El trabajo de campo se realizó entre marzo de 2017 y febrero de 2018.

\begin{tabular}{|c|c|}
\hline Información & Participantes $(n=22)$ \\
\hline \multicolumn{2}{|c|}{ Rango de edad } \\
\hline $25-34$ & 6 \\
\hline $35-44$ & 5 \\
\hline $45-54$ & 9 \\
\hline $55^{+}$ & 2 \\
\hline
\end{tabular}

Años de experiencia con mujeres con discapacidad

$<5$

$5-9$

$10-15$

$15-19$

$20^{+}$

Comunidad autónoma de experiencia $\left({ }^{*}\right)$

Madrid

Cataluña

Islas Baleares

Castilla y León

Andalucía

Aragón

Representante

Activista

Profesional

Investigadora

* Los datos muestran casos con representación múltiple, por ello no suma el total de participantes.

Se aplicaron 22 entrevistas semiestructuradas individuales en profundidad, cara a cara y a través de plataformas de comunicación online, con el fin de permitir la interacción con personas de distintas localizaciones geográficas. Todas fueron aplicadas por la investigadora principal, capacitada en la técnica y con experiencia de 11 años en investigación cualitativa. Los audios fueron grabados y transcritos de manera textual. Su duración promedio fue de 75 minutos. Como instrumento para recolectar la información se utilizó la entrevista episódica, mediante un guión temático con elementos narrativos, con el objeto de promover la narración de la informante sobre sus propias experiencias relacionadas a la detección de prácticas de vulneración de derechos sexuales y reproductivos hacia mujeres y/o niñas con discapacidad; prácticas de esterilización forzada hacia el colectivo; posturas personal e institucional frente a dichas prácticas; procedimientos para su ejecución.

El análisis siguió las estrategias de la Teoría Fundamentada Constructivista (Charmaz, 2006), iniciado con el levantamiento de categorías, finalizando en su integración y generación de la teoría integradora, un modelo explicativo relacional de los discursos. La elección de esta estrategia se debe a su pertinencia en investigaciones de procesos sociales que se han estudiado de manera insuficiente o con escasa profundidad y, a su vez, permite la comprensión teórica de un proceso situado (Charmaz, 2006). Para ello, dos investigadores, en forma independiente, analizaron por separado el material, utilizando el programa ATLAS.ti versión 8.4.4 (Friese, 2019). Con el apoyo de un tercer investigador se aseguró fiabilidad entre jueces. El número de participantes se determinó por criterio de saturación de datos, alcanzado con 18 entrevistas y se aplicaron 4 entrevistas más para corroborar hallazgos.

Como criterios de rigor se utilizaron la credibilidad, originalidad, resonancia y utilidad (Charmaz, 20o6). La credibilidad se alcanzó con triangulación teórica (revisión y discusión de estudios previos), de la codificación (3 analistas) y de investigadores (3 personas). En la descripción de resultados se informa confrontación de hallazgos, que avalan la coherencia estructural y de contenido. La originalidad al ofrecer un tratado original en un área con escaso desarrollo y mediante un profundo análisis del fenómeno estudiado. La resonancia se estableció mediante la descripción detallada de categorías e inclusión de citas directas de las 
entrevistas para ilustrar elementos para la toma de decisiones, logrado tras las reuniones de consenso del equipo investigador. Por último, la utilidad se vincula con que este análisis puede generar más investigación en áreas sustantivas, como en el ámbito de los derechos sexuales y reproductivos de este colectivo y al contribuir al desarrollo del conocimiento en el ámbito de violencia contra las mujeres. Con el fin de garantizar su calidad y transparencia se siguieron las normas de los Criterios Consolidados para Informes de Investigación Cualitativa (Tong; Sainsbury; Craig, 2007).

Los aspectos éticos fueron resguardados de acuerdo a los principios de la Declaración de Helsinki (WMA, 2013). Con cada participante se siguió el procedimiento de consentimiento informado, recibieron información del proyecto, sus objetivos, garantías de respeto a la autonomía, anonimato, confidencialidad. El proyecto fue evaluado y aprobado por el Comité de Ética de la Universidad donde se llevó a cabo el estudio.

\section{Resultados}

\section{Esterilización forzada actual}

La mayor parte de las informantes coinciden al distinguirla como una práctica de salud administrada específicamente a mujeres y niñas con discapacidad, cuya decisión es del entorno que rodea a la mujer o niña, aludiendo al motivo de su protección ante un posible embarazo y temor a reproducir la discapacidad. Enfatizan sobre la limitada visibilidad de la práctica ante la sociedad española, debido a su ocultamiento por parte de agentes involucrados en la práctica, y a la carencia de estadísticas y estudios que transparenten la vigencia del procedimiento a nivel nacional: Ha ocurrido siempre, hay muchas más de las que creemos. Como siempre, el mundo de la discapacidad era invisible hasta hace pocos años. Se ha ocultado este tema específico (E20, profesional).

Una minoría de profesionales difiere del resto de informantes acerca de la existencia actual de la práctica, ya sea porque manifiesta desconocer antecedentes o por no considerarlo como un tema 'forzoso'. Así lo relata una de ellas: No es forzosa, pero se recomienda a las personas afectadas por enfermedad mental tomar medidas anticonceptivas (E18, profesional).

Activistas e investigadoras destacan que esta práctica puede esconderse tras intervenciones ginecológicas destinadas a solucionar trastornos como endometriosis, dolores relacionados con la menstruación o cambios hormonales. Manifiestan que muchas veces se realizan mediante la entrega de información falsa respecto del procedimiento realizado o con coerción hacia las mujeres afectadas:

Muchas mujeres que se encuentran en esa situación de haber sido esterilizadas a la fuerza, realmente no saben que han sido esterilizadas, y, por otro lado, nadie les ha explicado qué significa que estén esterilizadas... los casos que conozco son personas que no saben que han sido esterilizadas y que les han dicho que han sido operadas de apendicitis o cualquier otra tontería y no saben qué significa la esterilización [...] las cifras son muy necesarias, porque no es algo puntual, es algo bastante generalizado. (E1, activista)

La mayoría de las participantes identifican circunstancias que ponen en mayor riesgo a la mujer o niña para ser sometida a esta práctica, como encontrarse en edad reproductiva, tener discapacidad intelectual y psicosocial, no obstante, se aplica a mujeres con todo tipo de discapacidades (física, intelectual, psíquica, sensorial, del espectro autista) con cualquier nivel de compromiso funcional (de leve hasta profundo), con altos niveles de dependencia y necesidad de asistencia, con nivel educativo mayoritariamente medio-bajo. No aprecian diferencia en el lugar de residencia (en una institución o con su núcleo familiar). La incapacitación judicial aplicada a la mujer es determinante, pero el elemento más decisivo visualizado es la confluencia de estas características mencionadas en la misma mujer:

Yo no me he encontrado un patrón de edad. Da exactamente igual si vive con familia o está institucionalizada. El número de mujeres institucionalizadas, aunque es más alto que el de hombres, son cifras relativamente bajas. 
Las mujeres que están institucionalizadas, normalmente, es porque la discapacidad ya tiene un grado de afectación muy alto y los casos de incapacitación son por casos de abusos muy concretos. Pero no hay un patrón. Es más, seguramente cuanta más libertad de movimiento tenga la mujer, más miedo tienen las familias que puedan quedarse embarazadas... Al final las prácticas lo que hacen es poner un parche a un montón de aspectos para los que no estamos preparados para dar inclusión. (E15, investigadora)

\section{Otras prácticas forzadas de regulación sexual y reproductiva relacionadas}

\section{Medidas de anticoncepción transitoria forzados}

La mayor parte de los relatos concuerdan en la existencia de estas medidas en forma más generalizada en la actualidad hacia el colectivo. De acuerdo con su visión, también son aplicadas de manera forzada. Las instituciones, profesionales y familiares no problematizan estas prácticas, al contrario, las justifican basándose en el carácter temporal de estas medidasy al proceso de incapacitación judicial establecido. Algunas profesionales expresan que con esta práctica no se da importancia al contagio de enfermedades de transmisión sexual o VIH y que favorece el encubrimiento de los abusadores. Muchas coinciden en reconocer a las familias como uno de los principales agentes que promueven estas prácticas:

Sé de muchas niñas que llevan el parche, o algún método de anticoncepción, que dure mucho tiempo, y que ellas no tengan ni que preocuparse, sabiendo que no todos los riesgos son el embarazo. Es que a las familias parece que el único riesgo que les importa es el embarazo, y no es el único riesgo. Hay otras infecciones que también son de bastante riesgo, pero sobre todo, el mayor riesgo es el psicológico, el que esa chica sea maltratada o que es a chica sea emocionalmente dañada, violada, perjudicada por hombres con o sin discapacidad y eso es un riesgo que no se asume. (E9, profesional)

Nuevamente aparece un grupo minoritario y transversal entre los grupos de informantes que manifiesta desconocer antecedentes sobre la existencia de este tipo de procedimientos.

\section{Otras prácticas de violencias asociadas}

Informantes de todos los grupos consultados identifican que las prácticas anteriores suelen coexistir junto a otros tipos de violencias, muchas arraigadas en las instituciones, como el quitar la tuición de hijos/as; la exposición y encubrimiento de violaciones y abusos reiterados; el no reconocimiento social de violencia de género contra estas mujeres y la carencia de atención a tales víctimas. En especial, activistas describen que estas prácticas forzadas y violencias asociadas afectan en mayor proporción a mujeres que conviven con más elementos de desigualdad, aunque aquellas con discapacidad, inmigrantes indocumentadas se percibe como uno de los grupos más violentados:

Haygran desprotecciónymuchomaltrato institucional. Por ejemplo, en el 2010 quedé embarazada y me despiden del trabajo. Llego al [institución de ayuda sociall y me exigen esterilizarme. Me quisieron quitar a mis hijos para darlos en adopción por estar en situación de inmigrante, indocumentada, sin trabajo y separada de un maltratador que estaba preso. Las instituciones en vez de ayudar, meten miedo [...] Conocí a una mujer brasileña en similar situación a la mía, que las instituciones la violentaron y persiguieron a tal nivel que llegó a un intento de suicidio y, luego de eso, más encima, la amenazaron con quitarle a sus hijos. (E21, activista)

Se agregan los abortos forzados, la privación de atención ginecológica accesible, la atención médica limitada por creencias y estereotipos de profesionales médicos/as y de salud, maternidades sin apoyos oficiales como asistencia personal o financiamientos, la privación de información sobre salud sexual y reproductiva accesible, no validar a la mujer exigiendo entregar información de carácter personal a un tercero y desprotección por mayor exposición a contagios por infecciones de transmisión sexual y VIH/SIDA:

Abortos coercitivos conozco muchos más. Esa sí que es una práctica común, o bien obligada por 
incapacitación, obligada por la presión del entorno, por médicos, familia [...] Por un lado, se le niega a la mujer ejercer su derecho a la sexualidad, y por otro, se les desprotege de posibles abusadores, dejándolas vulnerables. El estigma y la falta de información marcan sus historias vitales. (E15, investigadora)

Al igual que en la práctica anterior, un grupo minoritario y transversal entre los grupos de informantes niega su existencia o manifiesta desconocer antecedentes sobre estas violencias asociadas.

\section{Condiciones contextuales}

Para las informantes, el contexto en España al año 2017-2018, se caracteriza por el predominio de ciertos modelos que han permeado la estructura de las asociaciones y de las mismas personas con discapacidad, permitiendo prácticas de vulneración y de control de estas mujeres.

\section{Modelo patriarcal-capitalista-capacitista}

Surge principalmente de relatos de activistas e investigadoras. Desde su mirada, este modelo fomenta una ideología contra las mujeres, lo que se intensifica en aquellas con discapacidad. Visualizan que esta alianza de modelos criminaliza su deseo de maternidad, rechazando la reproducción de cuerpos considerados como no perfectos; defiende el dominio y control de sus cuerpos con intervenciones como las esterilizaciones, tras lo cual subyace la creencia de su inferioridad, anulación de su sexualidad, demostrando rechazo ante la diversidad humana por un ideal de perfección:

Para mí hay 3 ejes claves: el capacitismo, que es lo que censura estos cuerpos diversos y también en cierto modo el capitalismo, que tiene miedo de esta reproducción de los cuerpos que no son perfectos. Por otro, el patriarcado, porque esto se deposita en el cuerpo de la mujer, porque no hay una esterilización forzosa sistemática a hombres con diversidad funcional. O sea, por un lado, se las considera sexuales, pero por otro sigue primando una ideología en contra del género que hace que la responsabilidad recaiga en el cuerpo de la mujer, que es el que hay que guardar, que es el que hay que proteger, mientras que a los hombres no se les hace. Entonces es esa triple alianza. Una sociedad capacitista, capitalista y patriarcal. (E13, investigadora)

\section{Modelo médico-rehabilitador}

Distinguido por los 3 grupos consultados. Explican que su presencia actual es más predominante que los modelos sociales de la discapacidad. Declaran que fomenta la patologización, la devaluación y exclusión de estas mujeres, que sostiene la creencia de la curación como medio para la obtención de normalidad, que promueve la cultura del asistencialismo y caridad. Añaden que determina la anulación de la sexualidad en este colectivo, al no ser considerado como necesidad:

Porque consideran que tiene menos capacidad, porque su vida tiene menos valor, este valor está restringido a la posible curación y, si esa curación no se produce, tienen que quedar más apartados, ocultados. Esta es la historia de la concepción de la discapacidad, el modelo médico-rehabilitador [...] La base es la misma de cualquier tipo de discriminación, es que hay personas inferiores a otras, hay personas con menos valor que otras, por lo tanto, hay que 'protegerlas', porque es una falsa sensación de protección. (E22, activista)

\section{Condiciones facilitadoras}

\section{Instrumentalización de la incapacitación judicial}

Las informantes distinguen, transversalmente, que es una condición determinante de esta práctica en el país. Este elemento es reconocido como puerta de entrada al procedimiento de esterilización y a la muerte civil de estas mujeres. Se aprecia como una realidad generalizada y normalizada en el ámbito de la discapacidad, con el objeto de controlar las decisiones de estas mujeres:

Los padres también relatan cómo han esterilizado a las hijas principalmente. Ahí fue como darnos de bruces con una realidad muy generalizada y más bien la crítica de las familias iba en la línea de escollos judiciales, de que tienes que encontrar al juez o jueza que no te ponga ningún problema y te dé la validación para hacer este procedimiento... estas 
prácticas apoyan mucho la exclusión del colectivo, porque, al final, desexualizar es deshumanizar. (E11, investigadora)

Declaran que este procedimiento legal se asienta en relaciones jerárquicas, con asimetría de poder, donde se permite su abuso, sin reconocerlo como tal. Se da entre estas mujeres y los agentes involucrados en la decisión de ejecutar el procedimiento: Estado, instituciones, asociaciones, jueces, profesionales sanitarios y del ámbito socioeducativo, familias y/o representantes legales:

La última decisión debe ser del tutor legal. El Estado que lo permite, los profesionales que lo promueven y la familia que da el visto bueno. Ellos también se ven indefensos ante los profesionales y ante el Estado. Es el método para dar solución a estos problemas. (E6, profesional)

Especialmente activistas e investigadoras declaran que la incapacitación judicial en el país ha sido una vía para ejercer otros tipos de violencias hacia estas mujeres, tales como violencia sexual (protección a abusadores y exposición de la mujer), violencia psicológica (coerción, chantaje, sometimiento, quitar tuición de hijos, amenazas, anular autodeterminación), violencia económica (quitar patrimonio, generar dependencia económica), violencia institucional (muerte civil, quitar derecho a voto), entre otras:

Hay familias que utilizan la incapacitación con otros intereses, por cuestiones económicas, una manera de tener controlada a la persona, hay casos de matrimonios en proceso de divorcio y maridos amenazan a las esposas de incapacitarlas para quitarles a los hijos o el patrimonio. Hemos constatado que la familia sigue siendo el principal protector, pero también el principal foco de violencia. (E2, activista)

\section{Marcos legales a nivel nacional e internacional}

Recalcan la existencia de un marco internacional insuficiente en materia de discapacidad y derechos sexuales y reproductivos. Junto a ello, indican la existencia de leyes nacionales sin concienciación, con escasa priorización y presupuesto a nivel nacional, lo que dificulta su implementación. En el país reconocen la presencia de un código penal que vulnera derechos modificando capacidad legal de estas mujeres, avalando el procedimiento forzado de la esterilización:

Francamente me gustaría pensar que ha habido un antes y un después [sobre la creación de instrumentos legales sobre discapacidad], pero creo que no han tenido tanta relevancia, que todavía, sobre todo en personas incapacitadas legalmente, la familia toma las decisiones por ellas. Y no hay nadie que vele por esas decisiones si son o no consensuadas. (E14, investigadora)

\section{Violencia sexual}

Los 3 grupos de informantes recalcan la presencia de violencia sexual ejercida contra mujeres y niñas con discapacidad, señalando su alta frecuencia, pese a carecer de estadísticas oficiales. Los reconocen como delitos que no tienen consecuencias visibles, ocasionalmente solo demostradas por enfermedades de transmisión sexual, con una baja tasa de denuncias. Es una violencia que queda libre de detección y castigo para los abusadores. Reconocen que la institucionalización alberga con frecuencia este tipo de abusos:

Bueno eso ha habido en [nombre de institución] [...] se me ponen los pelos de punta, porque yo lo he vivido, síque estuve en un juicio de un auxiliar que mantuvo relaciones sexuales con una con retraso mental con problemas de conducta. (E1o, profesional)

Déficit educativo en derechos sexuales y reproductivos de mujeres con discapacidad

Identifican que el Estado no se ha hecho cargo de cubrir esta necesidad y que el abordaje del tema es desigual para la población española, debido a que la toma de decisiones es en forma aislada por cada comunidad autónoma, y cada asociación vinculada a discapacidad lo hace de igual forma. Perciben que estas mujeres y niñas quedan más expuestas al no tener herramientas educativas para enfrentar las esterilizaciones, resultando en mayor riesgo a contraer enfermedades de transmisión sexual, embarazos no deseados, abusos y vulneraciones de derechos en general: 
En ausencia de una buena formación pones a las niñas en el disparadero, las haces muy vulnerables de todo tipo de abusos. Nos afecta porque no estamos haciendo prevención de estos abusos, y más para las mujeres con discapacidad... Asísin duda alguna. Eso no va a cambiar su vulnerabilidad. Y quizá lo aumente, un blanco muy fácil. Solo se previene la consecuencia más inmediata. (E17, investigadora)

Advierten que profesionales vinculados a la atención de este grupo también desconocen estos temas, como se puede observar en el siguiente extracto:

En este centro [ocupacional] hay usuarios con incapacitación legal, pero no sé este tema cómo se maneja. Es un tema que no he entrado, porque muchas mujeres ven recortadas sus opciones sexuales. Este es un tema que no se toca porque nuestros usuarios no tienen relaciones sexuales. (E8, profesional)

\section{Modelo explicativo-relacional de los discursos}

Como etapa final del análisis, surge el modelo de prácticas de salud de dominación y exclusión de mujeres y niñas con discapacidad (Figura 1). Este modelo sugiere que el fenómeno central de esterilización forzada en la sociedad actual española, llevado a cabo junto a medidas de anticoncepción transitoria forzada y otras prácticas de violencia asociadas, se distingue por las informantes como prácticas de salud diferenciadas, dirigidas a personas consideradas socialmente diferentes, pues ante la exposición de toda la población a un problema social como la violencia sexual, este tipo de intervención es privativa del colectivo de mujeres y niñas con discapacidad y, con mayor magnitud, en quienes confluyen diversos ejes de desigualdad, como se observó en mujeres con discapacidad, migrantes e indocumentadas. Hacia el colectivo se han instaurado intervenciones fundamentadas en las problemáticas reveladas en torno a su salud sexual y reproductiva, sin embargo, en lugar de solucionarlas, las han agravado y profundizado. Las medidas implementadas, a través de procesos de medicalización y control de sus cuerpos inferiorizados, han controlado y condicionado su salud sexual y reproductiva, han dejado a las mujeres expuestas a un mayor riesgo de esta y otras violencias y han promovido mecanismos de exclusión social e inequidades en salud y bienestar, al privarles de sus derechos humanos fundamentales.

\section{Figura I - Modelo de prácticas de salud de dominación y exclusión de mujeres y niñas con discapacidad}

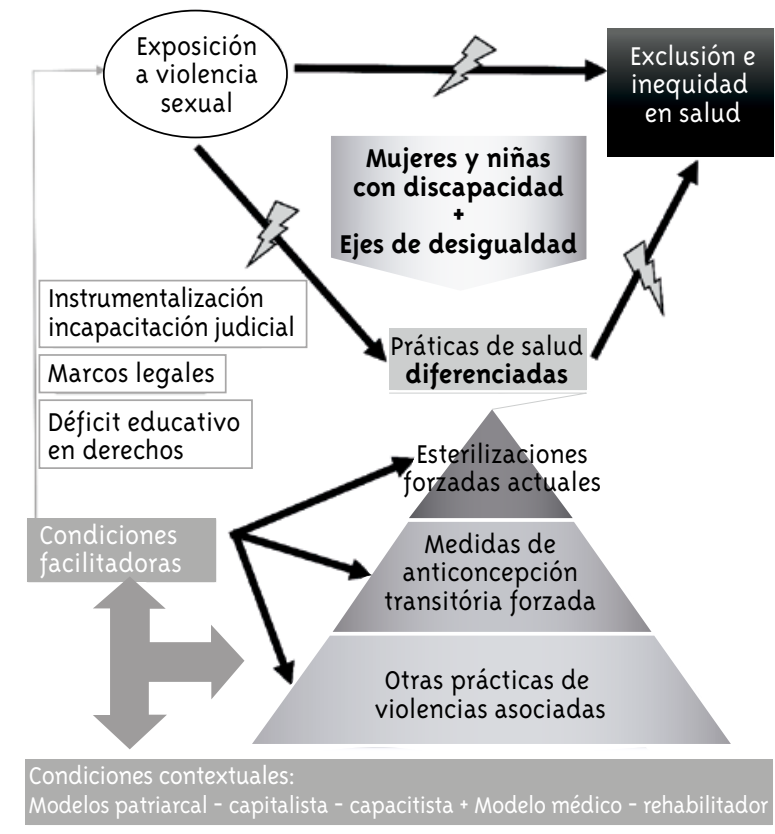

\section{Discusión}

El objetivo general de este estudio fue presentar un análisis sobre la situación de la práctica de esterilización forzada en mujeres y niñas con discapacidad en España, a partir de los discursos de mujeres representativas vinculadas a este colectivo. De acuerdo a su visión, la sexualidad y la reproducción de las mujeres con discapacidad en el país ha sido objeto de expropiación, alienación y desprecio por parte de sistemas, estructuras y políticas, con escasa transferencia del marco global de derechos humanos a la realidad cotidiana del colectivo. Mientras, la violencia sexual ha pasado inadvertida, naturalizándose por el entorno con el uso de intervenciones que no se han centrado en quienes cometen y continúan ejerciendo la violencia sexual permaneciendo impunes, sino por el contrario, enfocándose en las mujeres y niñas agredidas, mediante la limitación de su capacidad 
reproductiva. Lo anterior coincide con quienes plantean que la salud pública se ha centrado en cambiar los comportamientos en lugar de cambiar los entornos en los cuales los comportamientos son necesarios para sobrevivir y los enfoques de intervención a menudo demuestran una tendencia a culpar a las víctimas (Castle et al., 2019).

Estos hallazgos permiten visualizar la presencia de dinámicas de exclusión que operan en diversos ámbitos (salud, educación, social, legal, judicial) demostrando la existencia de racionalidades basadas en la jerarquización de las diversas formas de desigualdad (género, discapacidad y edad en interacción con determinantes sociales tales como pertenencia a un grupo étnico, acceso limitado a información, condiciones de pobreza, de inmigración, marginalidad, entre otras), que justifican la generación de políticas que reproducen la desigualdad, materializándose en prácticas de salud de dominación y exclusión estudiadas. Se observa que estas constituyen el último eslabón de un entramado de lógicas antagónicas que subyacen a sus condiciones contextuales y facilitadoras. Esa compresión dicotómica de las prácticas se aprecia a través de múltiples dimensiones de los discursos: reconocimiento $\mathrm{v} / \mathrm{s}$ no reconocimiento, que van desde un reconocimiento de la aplicación de procedimientos forzados hasta su no reconocimiento o uso de la connotación 'forzada', como prestaciones de salud aceptadas; destrucción v/s preservación, se justifica la destrucción de la capacidad sexual y reproductiva, bajo el propósito de preservar la integridad de la persona y la de su familia; dimensión abandono v/s protección, donde la exposición de estas mujeres y niñas a violencia sexual se protege mediante soluciones institucionales que las dejan en completo abandono. No obstante, de acuerdo con la situación analizada, estas contradicciones se inclinan a favor del no reconocimiento, destrucción, abandono y, por tanto, exclusión social. De acuerdo al planteamiento de Santos (2007) y Oliveira (2018), esta situación se ajusta a lo definido como manifestación del racismo, elemento que, al ser utilizado como dispositivo biopolítico, colabora en la comprensión de políticas diferenciadas acorde a diversas divisiones económicas, sociales, étnicas, de género, entre otras. Es decir, el racismo como un determinante social de salud, actuando a través de prácticas discriminatorias en una población marginada (Castle et al., 2019). Estos hallazgos son consistentes con lo planteado por la literatura que denuncia inequidad en el acceso a los servicios de salud por parte de la población con discapacidad en España, mayormente percibida por mujeres (Hernández; Jiménez, 2011); y por estudios que plantean la atribución de escaso valor social a quienes son excluidos socialmente, se les niega el disfrute de oportunidades disponibles para otros, incluyendo el disfrute de una buena salud (Tamayo; Besoaín; Rebolledo, 2018).

Las limitaciones de este estudio apuntan a sus dificultades para la generalización de sus resultados, de acuerdo a sus características cualitativas y a la alta tasa de rechazo de las participantes invitadas, lo que implica el continuar investigando y profundizando las temáticas aquí expuestas, como los derechos sexuales y reproductivos de este colectivo y contribuir al desarrollo del conocimiento en el ámbito de violencia contra las mujeres. Sin embargo, como trata de una situación que se replica en otros lugares del mundo, debiese considerarse un tema de relevancia para la salud pública internacional. Su fortaleza radica en su contribución a cubrir el vacío de investigación existente sobre el tema, y difundir este conocimiento colabora en concientizar sobre una práctica de salud considerada como un tipo violencia contra las mujeres y niñas escasamente visible que se produce a nivel mundial. La importancia de estos hallazgos implica a profesionales de salud, instituciones y tomadores de decisiones de distintas latitudes, quienes tienen la obligación de avanzar hacia la erradicación de prácticas de salud de violencia contra las mujeres y promoción de prácticas más equitativas y justas socialmente.

\section{Referencias}

ALBERT, G.; SZILVASI, M. Intersectional discrimination of Romani women forcibly sterilized in the former Czechoslovakia and Czech Republic. Health and Human Rights, Boston, v. 19, n. 2, p. 23-34, 2017.

ARNAU, M. S. Crímenes olvidados: el holocausto y las personas con diversidad funcional. In: ABFyC - 
ASOCIACIÓN DE BIOÉTICA FUNDAMENTAL Y CLÍNICA. La bioética y el arte de elegir. 2. ed. Madrid, 2014. p. 462-473.

BIGLIA, B.; VERGÉS-BOSCH, N. Cuestionando la perspectiva de género en la investigación. Reire: Revista d'Innovació i Recerca en Educació, Barcelona, v. 9, n. 2, p. 12-29, 2016.

CASTLE, B. et al. Public health's approach to systemic racism: a systematic literature review. Journal of Racial and Ethnic Health Disparities, Washington, DC, v. 6, n. 1, p. 27-36, 2019.

CERMI - FUNDACIÓN CERMI MUJERES; EDF FORO EUROPEO DE LA DISCAPACIDAD. Poner fin a la esterilización forzosa de las mujeres y niñas con discapacidad. Madrid: Cinca, 2017.

CHARMAZ, K. Constructing grounded theory: a practical guide through qualitative analysis. Los Angeles: Sage, 2006.

CONSEJO DE EUROPA. Convenio del Consejo de Europa sobre prevención y lucha contra la violencia contra las mujeres y la violencia doméstica. Estambul, 2011. (Council of Europe Treaty Series, n. 210). Disponível em: <https://bit.ly/35WNonK>. Acesso em: 8 dez. 2020. ESPAÑA. Ley Orgánica n ${ }^{0}$ 3, de 21 de junio de 1989. De actualización del Código Penal. Boletín Oficial del Estado, Madrid, 22 jun. 1989. p. 8.

ESPAÑA. Ley Orgánica ${ }^{0}$ 10, de 23 de noviembre de 1995. Del Código Penal. Boletín Oficial del Estado, Madrid, 24 nov. 1995. p. 72.

FRIESE, S. Qualitative data analysis with ATLAS. ti. London: Sage, 2019.

HERNÁNDEZ, C.; JIMÉNEZ, D. Inequidad en la utilización de servicios sociosanitarios en España para las personas discapacitadas. Gaceta Sanitaria, Barcelona, v. 25, p. 85-92, 2011. Suplemento 2.

OHCHR - OFFICE OF THE HIGH COMMISSIONER FOR HUMAN RIGHTS et al. Eliminating forced, coercive and otherwise involuntary sterilization: an interagency statement. Geneva: WHO, 2014.

OLIVEIRA, R. G. Práticas de saúde em contextos de vulnerabilização e negligência de doenças, sujeitos e territórios: potencialidades e contradições na atenção à saúde de pessoas em situação de rua. Saúde e Sociedade, São Paulo, v. 27, n. 1, p. 37-50, 2018.

ONU - NACIONES UNIDAS. Estatuto de Roma de la Corte Penal Internacional. Roma, 2003. Disponível em: <https://bit.ly/2M51Xgv>. Acesso em: 7 dez. 2020.

ONU - NACIONES UNIDAS. Comité para la Eliminación de la Discriminación contra la Mujer. Lista de cuestiones y preguntas previas a la presentación del noveno informe periódico de España. Nueva York, 2019a.

ONU - NACIONES UNIDAS. Comité sobre los Derechos de las Personas con Discapacidad. observaciones finales sobre los informes periódicos segundo y tercero combinados de España. Nueva York, 2019b.

ROWLANDS, S.; WALE, J. Sterilisations at delivery or after childbirth: addressing continuing abuses in the consent process. Global Public Health, New York, v. 14, n. 8, p. 1153-1166, 2019.

SANTOS, B. S. Para além do pensamento abissal: das linhas globais a uma ecologia de saberes. Novos Estudos Cebrap, São Paulo, n. 79, p. 71-94, 2007.

SIFRIS, R. The involuntary sterilisation of marginalised women: power, discrimination, and intersectionality. Griffith Law Review, Queensland, v. 25, n. 1, p. 45-70, 2016.

STAKE, R. Investigación con estudio de casos. Madrid: Morata, 1999.

STANSFIELD, A. J.; HOLLAND, A. J.; CLARE, I. C. $H$. The sterilisation of people with intellectual disabilities in England and Wales during the period 1988 to 1999. Journal of Intellectual Disability Research, London, v. 51, n. 8, p. 569-579, 2007.

STERILIZATION of women and girls with disabilities: a briefing paper. Human Rights Watch, Washington, DC, 10 nov. 2011. Disponível em: <https://bit.ly/2XZL2OS>. Acesso em: 20 jan. 2021.

TAMAYO, M.; BESOAÍN, Á.; REBOLLEDO, J. Determinantes sociales de la salud y discapacidad: 
actualizando el modelo de determinación. Gaceta Sanitaria, Barcelona, v. 32, n. 1, p. 96-100, 2018.

TONG, A.; SAINSBURY, P.; CRAIG, J. Consolidated criteria for reporting qualitative research (Coreq): a 32-item checklist for interviews and focus groups. International Journal for Quality in Health Care, Dublin, v. 19, n. 6, p. 349-357, 2007.

UN - UNITED NATIONS. Convention on the rights of persons with disabilities. New York, 2007. Disponível em: <https://bit.ly/3ooyvWo>. Acesso em: 5 jan. 2020.

UN - UNITED NATIONS. Sexual and reproductive health and rights of girls and young women with disabilities. New York, 2017. Disponível em: <https://bit.ly/30XlIF8>. Acesso em: 5 jan. 2020.

UNHRC - UNITED NATIONS HUMAN RIGHTS COUNCIL. Promotion and protection of all human rights, civil, political, economic, social and cultural rights, including the right to development: report of the Special Rapporteur on torture and other cruel, inhuman or degrading treatment or punishment, Manfred Nowak. New York: UN, 2008. Disponível em: <https://bit.ly/3pзUBYf >. Acesso em: 6 jan. 2020.

UN WOMEN - UNITED NATIONS ENTITY FOR GENDER EQUALITY AND THE EMPOWERMENT OF WOMEN. Fourth World Conference on Women. New York: ONU, 1996. Disponível em: <https://bit.ly/ 2LRMP6j>. Acesso em: 7 jan. 2020.

WMA - WORLD MEDICAL ASSOCIATION. World Medical Association Declaration of Helsinki: ethical principles for medical research involving human subjects. Jama, Chicago, v. 310, n. 20, p. 2191-2194, 2013.

YUPANQUI, A.; FERRER, V. A. Análisis de la producción científica mundial sobre esterilización forzada de mujeres con discapacidad entre 1997 y 2016. Gaceta Sanitaria, Barcelona, v. 33, n. 4, p. 381-388, 2019.

\section{Agradecimientos}

Gracias a todas las mujeres que aceptaron participar en este estudio, pues gracias a ustedes es posible hacer visibles estas violencias. Este estudio fue realizado en el contexto de los estudios doctorales de la primera autora, los que han sido financiados gracias al apoyo de CONICYT-Becas Chile/Folio 72160267 y a la Universidad de Magallanes.

\section{Contribución de los autores}

Yupanqui-Concha y Ferrer-Perez fueron responsables de la concepción y diseño del estudio. La recolección de datos fue realizada por Yupanqui-Concha. Todo el equipo autoral contribuyó en los análisis de datos, en la discusión teórica de los resultados y en la elaboración del manuscrito final.

Recibido: 21/02/2020

Aprobado: 09/09/2020 